\title{
Enthalpies of Formation of Squaric Acid and the Corresponding Diethyl Ether
}

\author{
PETER SELLERS
}

Thermochemistry Laboratory, Chemical Center, University of Lund, S-220 07 Lund 7, Sweden

\begin{abstract}
Enthalpies of combustion have been measured for squaric acid (dihydroxycyclobutenedione) and diethoxycyclobutenedione. The following enthalpies of formation were derived:

$$
\begin{array}{ll} 
& \Delta H_{\mathrm{f}}^{\circ} / \mathrm{kJ} \mathrm{mol}^{-1} \\
\text { Squaric acid (c) } & -598.2 \pm 0.4 \\
\text { Diethoxycyclobutenedione (l) } & -552.1 \pm 1.4
\end{array}
$$

Using estimated values of enthalpies of sublimation and vaporization, the values are discussed in terms of strain energies and delocalization energies. The strain energy in a four-membered ring with four $s p^{2}$. hybridized carbon atoms is discussed.
\end{abstract}

$\mathrm{D}_{\mathrm{s}}^{\mathrm{i}}$ iketocyclobutenediol (3,4-dihydroxy-cyclobut-3-ene-1,2-dione) was first synthesized in $1959,{ }^{1}$ and was later given the trivial name squaric acid. ${ }^{2}$ The compound was found to be a strong acid, and the dianion was later shown to be a member of a new class of aromatic compounds, $\mathrm{C}_{n} \mathrm{O}_{n}^{-m}$, symmetrical resonance stabilized anions. ${ }^{3}$ The name oxocarbons ${ }^{4}$ was suggested for this class of compounds, in which all, or nearly all, of the carbon atoms bear ketonic functions or their equivalents.

Compounds possessing the cyclobutadienoquinone structure have been the subject of many recent investigations, since they are molecules which should have the same degree of strain as cyclobutadiene, which has not yet been isolated. Thermochemical data have been determined so far for only one member of this class of compound, phenylcyclobutenedione, ${ }^{5}$ and the present work was carried out to provide more information on such systems. The diethyl ether derivative of squaric acid was included to avoid the hydrogen bonding effects which exist in the acid itself.

\section{EXPERIMENTAL}

Compounds. Squaric acid (Fluka) was purified following the procedure given in Ref. 2, the recrystallization process being repeated 3 times. The compound was dried at $120^{\circ} \mathrm{C}$ and stored in a desiccator over $\mathrm{P}_{2} \mathrm{O}_{5}$. The water content of the sample was determined, using a coulometric Karl Fischer method, ${ }^{6}$ and found to be 0.003 wt. $\%$. The density of the sample was $2.119 \mathrm{~g} \mathrm{ml}^{-1}$ at $25^{\circ} \mathrm{C}$.

Acta Chem. Scand. 25 (1971) No. 6 
1,2-Diethoxycyclobutenedione (Fluka, pract.) was purified by distillation under reduced pressure, and then dried over molecular sieves $4 \mathrm{~A}$. The purity of the sample was checked by GLC on a SE-30 column. No organic impurities were detected. The water content of the sample was determined on ampoules filled under vacuum, using a gas chromatographic method, ${ }^{7}$ and found to be $0.007 \mathrm{wt} . \%$. The density of the sample was $1.1588 \mathrm{~g} \mathrm{ml}^{-1}$ at $25^{\circ} \mathrm{C}$.

Combustion calorimetry - apparatus and procedure. The apparatus and calorimetric procedure were as described previously. ${ }^{8}$ The squaric acid sample was pressed into pellets for the combustion experiments. It was not found necessary to enclose the pellets; the substance has a very low vapour pressure at $25^{\circ} \mathrm{C}$, and is not hygroscopic (no significant weight changes after $1 / 2 \mathrm{~h}$ on a microbalance).

The diethoxycyclobutenedione was filled into soda-glass ampoules under vacuum.

Enthalpies of vaporization and sublimation. No experimental values could be obtained at the present time. Instead, calculated values were derived, using a group-incremental method.

\section{RESULTS}

The results are expressed in terms of absolute joules. Symbols and calculational procedure used were as given previously.$^{8,9}$ All weighings were reduced to mass, and the molecular weights computed from the 1969 Table of Atomic Weights. ${ }^{10}$ The values used for the specific heat capacities and $(\delta V / \delta T)_{\mathrm{p}}$ for squaric acid and the diethoxy compound were $1.40 \mathrm{~J} \mathrm{~K}^{-1} \mathrm{~g}^{-1}$ and $0.400 \mathrm{~mm}^{3}$ $\mathrm{K}^{-1} \mathrm{~g}^{-1}$, and $1.67 \mathrm{~J} \mathrm{~K}^{-1} \mathrm{~g}^{-1}$ and $0.646 \mathrm{~mm}^{3} \mathrm{~K}^{-1} \mathrm{~g}^{-1}$, respectively.

Auxiliary data used and estimation of the final over-all precision were as previously. ${ }^{8,9}$ Data for a typical combustion experiment for each compound

Table 1. Summary of typical combustion experiments.

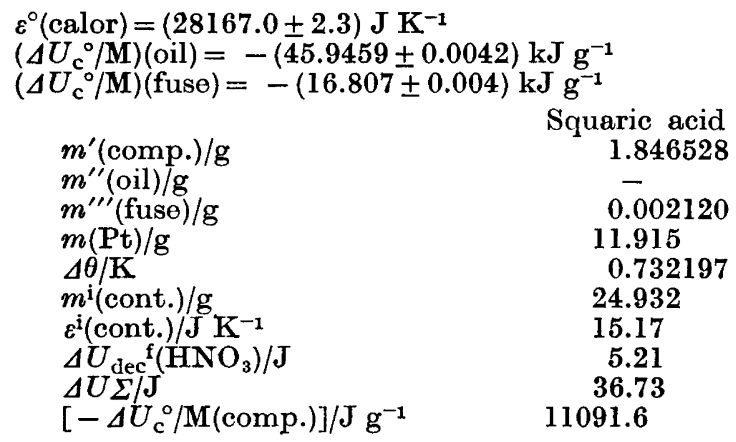

Diethoxycyclobutenedione 0.514665

0.176886

0.001277

11.915

0.724204

23.837

13.88

7.65

11.02

23645.0

Table 2. Results of combustion experiments at $25^{\circ} \mathrm{C}$.

$\begin{array}{lcc} & -\left(\Delta U_{\mathrm{c}}^{\circ} / \mathrm{M}\right) \mathrm{kJ} \mathrm{g}^{-1} & -\left(\Delta U_{\mathrm{c}}{ }^{\circ} / \mathbf{M}\right) \mathrm{kJ} \mathrm{g}^{-1} \\ & \text { Squaric acid } & \text { Diethoxycyclobutenedione } \\ & 11.0967 & 23.6508 \\ & 11.0961 & 23.6450 \\ & 11.0921 & 23.6413 \\ & 11.0916 & 23.6507 \\ & 11.0932 & 23.6378 \\ \text { Mean } & 11.0949 & 23.6451 \\ \text { Standard deviation } & 11.0941 & 0.0026 \\ & 0.0009 & -\Delta U_{\mathrm{c}}^{\circ} / \mathbf{M}=\mathbf{2 3 . 6 4 6 8} \mathbf{k J ~ g}^{-1}\end{array}$

Acta Chem. Scand. 25 (1971) No. 6 
PETER SELLER S
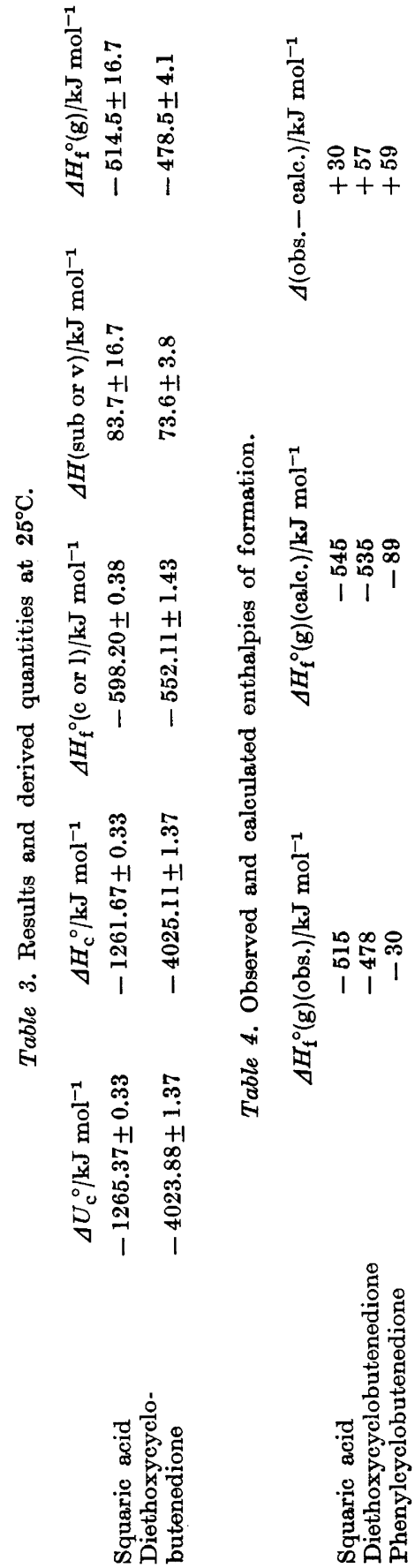

Acta Chem. Scand. 25 (1971) No. 6 
are given in Table 1. The results from the different series of measurements are summarized in Table 2, where the given uncertainties are the standard deviations of the means. The $\Delta U_{\mathrm{c}}^{\circ} / \mathrm{M}$ mean value for diethoxycyclobutenedione given in Table 2 refers to the pure sample after correction for the water content.

Table 3 gives the standard energies, $\Delta U_{\mathrm{c}}^{\circ}$, and enthalpies, $\Delta H_{\mathrm{c}}^{\circ}$, of combustion, together with the estimated enthalpies of vaporization and sublimation, $\Delta H_{\mathrm{v}}$ and $\Delta H_{\mathrm{sub}}$, and derived enthalpies of formation, $\Delta H_{\mathrm{f}}^{\circ}$, for the liquid or solid and gaseous states. The errors given are twice the final over-all standard deviation of the mean, except for $\Delta H_{\mathrm{v}}, \Delta H_{\text {sub }}$ and $\Delta H_{\mathrm{f}}^{\circ}(\mathrm{g})$, where estimated uncertainties in the calculated values are included.

\section{DISCUSSION}

The enthalpies of vaporization and sublimation calculated for the compounds are associated with fairly large errors, especially in the case of squaric acid, where hydrogen bonding in the crystal ${ }^{11}$ makes the estimation particularly difficult. These errors, however, will not be as serious as would normally be the case, since, as will be seen below, uncertainties associated with the reference systems are also high, and only a qualitative picture of the effects under investigation can be obtained.

In order to derive information regarding strain and delocalization energies in the molecules, it is necessary to calculate values for a reference system. No data are available for suitable reference compounds, and the bond energy schemes usually most satisfactory for the calculation of reference data 12 are not applicable in the present case; parameters for some of the bonds and interactions are not known. It has therefore been necessary to resort to a simpler scheme, namely that of Klages, ${ }^{13}$ which enables the calculation of the enthalpy of combustion, and thus enthalpy of formation, in the vapour state of the strain-free and non-resonating molecules. This method was also used by Roberts and co-workers ${ }^{5}$ in the case of phenylcyclobutenedione, so that some uniformity in the method of approach will be maintained.

Table 4 shows the experimental and calculated enthalpies of formation for the compounds concerned, with the data for the above-mentioned phenyl compound included for comparison. The last column gives the difference between the observed and calculated values, where a correction has been made in the case of the phenyl compound for the delocalization energy of the phenyl group, $151 \mathrm{~kJ} \mathrm{~mol}^{-1}$. The results indicate that for all three compounds, the strain energy of the four-membered ring exceeds the delocalization energy of the ring, and, considering the uncertainties in the corrections to the vapour state and calculations on reference systems, the results are in good agreement.

In the paper where the results for the phenyl compound were originally presented, ${ }^{5}$ Roberts deduced that the delocalization energy in the fourmembered ring would be between about 130 and $250 \mathrm{~kJ} \mathrm{~mol}^{-1}$. This was based on theoretical calculations of the strain energy in cyclobutadiene, ${ }^{14,15}$ which gave the strain energy as being between 190 and $310 \mathrm{~kJ} \mathrm{~mol}^{-1}$, and assuming the ring strain in the dione to be the same. Further support for a high value of the strain energy in a four-membered ring with four trigonal carbon atoms is provided by the work of Bedford et al., ${ }^{16}$ who deduced a strain

Acta Chem. Scand. 25 (1971) No. 6 
energy of the four-membered ring of biphenylene of between 270 and 315 $\mathrm{kJ} \mathrm{mol}^{-1}$, using a comparison with biphenyl. ${ }^{17}$ In a footnote in Ref. 5, Roberts implied that perhaps the relevant strain energy should be much lower, and in other publications ${ }^{18,19}$ he has suggested a value of between 75 and 126 $\mathrm{kJ} \mathrm{mol}$ "1, that is, of about the same order as that in a "normal" four-membered ring. If this is the case, then the delocalization energy of the systems given in Table 4 would be of the order of $40 \mathrm{~kJ} \mathrm{~mol}^{-1}$. There have been some theoretical calculations of the delocalization energies in such systems, using a simple LCAO approach. ${ }^{5,20}$ These calculations indicate a delocalization energy in the parent cyclobutadienoquinone of about $90 \mathrm{~kJ} \mathrm{~mol}^{-1}$, and in the phenyl compound of about $125 \mathrm{~kJ} \mathrm{~mol}^{-1}$. These values, combined with the data of Table 4, support the high figure for the strain energy in the rings under study, but too much weight should not be put on them, since they are crude approximations.

The situation is thus rather confused, and no definite conclusions can be drawn. The present author is inclined to the view that the strain energy in question should be considerably higher than that in cyclobutane, but not as high as that quoted for the ring in biphenylene. A more definite answer to the problem can be obtained only when theoretical calculations can be refined and data obtained for molecules studied, which are uncompromised by effects other than strain.

Acknowledgements. The author is indebted to Dr. Ernst Morawetz for help with the. calculations on enthalpies of sublimation and vaporization.

\section{REFERENCES}

1. Cohen, S., Lacher, J. R. and Park, J. D. J. Am. Chem. Soc. 81 (1959) 3480.

2. Park, J. D., Cohen, S. and Lacher, J. R. J. Am. Chem. Soc. 84 (1962) 2919.

3. West, R., Niu, H. Y., Powell, D. L. and Evans, M. W. J. Am. Chem. Soc. 82 (1960) 6204.

4. West, R. and Powell, D. L. J. Am. Chem. Soc. 85 (1963) 2577.

5. Smutny, E. J., Caserio, M. C. and Roberts, J. D. J. Am. Chem. Soc. 82 (1960) 1793.

6. Karlsson, R. and Karrman, K. J. Talanta 18 (1971) 459.

7. Sellers, P. Acta Chem. Scand. 25 (1971) 2295.

8. Sellers, P. Acta Chem. Scand. 25 (1971) 2099.

9. Mansson, M. J. Chem. Thermodynamics 1 (1969) 141.

10. Comptes Rendus of the XXVth IUPAC Conference 1969, p. 103.

11. West, R. and Niu, J. In Snyder, J. P., Ed., Nonbenzenoid Aromatics I, Academic, New York 1970, Chap. 6.

12. Cox, J. D. and Pilcher, G. Thermochemistry of Organic and Organometallic Compounds, Academic, London 1970, Chap. 7.

13. Klages, F. Chem. Ber. 82 (1949) 358.

14. Weltner, Jr., W. J. Am. Chem. Soc. 75 (1953) 4224.

15. Coulson, C. A. and Moffitt, W. E. Phil. Mag. 40 (1969) 1.

16. Bedford, A. F., Carey, J. G., Millar, I. T., Mortimer, C. T. and Springall, H. D. J. Chem. Soc. 19623895.

17. Parks, G. S. and Vaughan, L. M. J. Am. Chem. Soc. 73 (1951) 2380.

18. Krall, R. E. and Roberts J. D. Am. Chem. Soc., Div. Petrol. Chem. Symp. 3, B 4 (1958) 63.

19. Baker, W. and McOmie, J. F. W. In Ginsburg, D., Ed., Nonbenzenoid Aromatic Compounds, Interscience, New York 1959, Chap. 2.

20. Cava, M. P. and Mitchell, M. J. Cyclobutadiene and Related Compounds, Academic, New York 1967, Chap. 4.

Received October 29, 1970.

Acta Chem. Scand. 25 (1971) No. 6 